\title{
Decitabine Maintains Hematopoietic Precursor Self-Renewal by Preventing Repression of Stem Cell Genes by a Differentiation-Inducing Stimulus
}

Zhenbo $\mathrm{Hu}^{1}$, Soledad Negrotto ${ }^{1}$, Xiaorong $\mathrm{Gu}^{1}$, Reda Mahfouz ${ }^{1}$, Kwok Peng $\mathrm{Ng}^{1}$, Quteba Ebrahem ${ }^{1}$, Edward Copelan ${ }^{2}$, Harinder Singh ${ }^{3}$, Jaroslaw P. Maciejewski ${ }^{1,2}$, and Yogen Saunthararajah ${ }^{1,2}$

Abstract

The cytosine analogue decitabine alters hematopoietic differentiation. For example, decitabine treatment increases self-renewal of normal hematopoietic stem cells. The mechanisms underlying decitabine-induced shifts in differentiation are poorly understood, but likely relate to the ability of decitabine to deplete the chromatin-modifying enzyme DNA methyltransferase 1 (DNMT1), which plays a central role in transcription repression. HOXB4 is a transcription factor that promotes hematopoietic stem cell self-renewal. In hematopoietic precursors induced to differentiate by the lineage-specifying transcription factor Pu. 1 or by the cytokine granulocyte-colony stimulating factor, there is rapid repression of HOXB4 and other stem cell genes. Depletion of DNMT1 using shRNA or decitabine prevents HOXB4 repression by Pu.1 or granulocyte-colony stimulating factor and maintains hematopoietic precursor self-renewal. In contrast, depletion of DNMT1 by decitabine 6 hours after the differentiation stimulus, that is, after repression of HOXB4 has occurred, augments differentiation. Therefore, DNMT1 is required for the early repression of stem cell genes, which occurs in response to a differentiation stimulus, providing a mechanistic explanation for the observation that decitabine can maintain or increase hematopoietic stem cell self-renewal in the presence of a differentiation stimulus. Using decitabine to deplete DNMT1 after this early repression phase does not impair progressive differentiation. Mol Cancer Ther; 9(6); 1536-43. (2010 AACR.

\section{Introduction}

The cytosine analogue 5-aza-2'-deoxycytidine (decitabine), approved by the U.S. Food and Drug Administration as a treatment for myelodysplastic syndrome, alters hematopoietic differentiation. Therapeutically important differentiation-altering effects of decitabine include terminal differentiation of leukemia cells $(1-5),{ }^{4}$ increased erythropoiesis and fetal hemoglobin to treat sickle cell disease and $\beta$-thalassemia $(6,7)$, and increased selfrenewal of hematopoietic stem cells, which could have a role in ex vivo expansion of hematopoietic stem cells (8-10). The mechanisms underlying these decitabineinduced shifts in differentiation are poorly understood, but likely relate to the ability of decitabine to deplete the chromatin-modifying enzyme DNA methyltransfer-

\footnotetext{
Authors' Affiliations: Departments of 1 Translational Hematology and Oncology Research and 2 Hematologic Oncology and Blood Disorders, Taussig Cancer Institute, Cleveland Clinic, Cleveland, Ohio and ${ }^{3}$ Department of Molecular Genetics and Cell Biology, University of Chicago, Chicago, Illinois

Note: Z. Hu and S. Negrotto contributed equally to this work.

Corresponding Author: Yogen Saunthararajah, Taussig Cancer Institute, 9500 Euclid Avenue R40, Cleveland, OH 44195. Phone: 216-444-8170; Fax: 216-636-2498. E-mail: saunthy@ccf.org

doi: 10.1158/1535-7163.MCT-10-0191
}

(C2010 American Association for Cancer Research. ase 1 (DNMT1; ref. 11). DNMT1 duplicates methylation marks onto the newly synthesized DNA strand during mitosis (maintenance methylation; ref. 12). DNMT1 is also a component of histone methyltransferase protein complexes that create methylation marks of transcription repression (reviewed in ref. 13) and histone demethylase protein complexes that remove methylation marks of transcription activation (14). Accordingly, decitabine treatment to deplete DNMT1 not only hypomethylates DNA but can also extensively modify chromatin, producing decondensation of chromatin structure (15).

Drugs that relax chromatin by inhibiting histone deacetylase (HDAC) produce changes in hematopoietic differentiation similar to that produced by decitabine: terminal differentiation of leukemia cells $(4,16,17)$, increased fetal hemoglobin expression (reviewed in ref. 18), and increased normal hematopoietic stem cell self-renewal $(8,19,20)$. Because a shared property of HDAC inhibitors and DNMT1 depletion by decitabine is antagonism of transcription repression, this suggests that antagonism of transcription repression could have a mechanistic role in producing the observed shifts in differentiation by decitabine and by HDAC inhibitors.

\footnotetext{
${ }^{4}$ Submitted for publication.
} 
The transition from one differentiation level to the next likely involves repression of key genes that maintain the initial or starting differentiation level. Here, we examined the possibility that decitabine antagonizes the repression of stem cell-associated genes by a differentiationinducing stimulus. We used two in vitro models of hematopoiesis to examine this possibility: a model of lineage-specifying transcription factor (Pu.1)-driven differentiation of murine hematopoietic cells and a model of cytokine [granulocyte-colony stimulating factor (G-CSF)]-driven differentiation of human hematopoietic cells. The generated observations provide a mechanistic explanation for some of the differentiation-altering effects of decitabine treatment and can guide the application of this drug.

\section{Materials and Methods}

\section{Generation of PUER cells with stable suppression of Dnmt1 expression}

A lentiviral vector, pLenti6-DEST (Invitrogen), was used to construct short hairpin (sh) RNA for Dnmt1. The specific 21-bp target sequences for mouse shDnmt1 (5'-GAACGGCATCAAGGTGAAC-3') were synthesized in sense and antisense orientations by Advanced DNA Technology; the single-strand oligos were then annealed to form double-strand oligos and subsequently ligated with pENTRY vector (Invitrogen) downstream of an RNA promoter. The ligated constructs were transformed into E. coli TOPO10. Positive clones were verified by DNA sequencing. The verified clones were then recombined into pLenti6-DEST vector using the Invitrogen ViralPack kit, resulting in pLenti6-Dnmt1. The pLenti6Dnmt1 constructs were then transfected together with envelop-encoding plasmid (VSVG) into 293FT packaging cell line to produce lentivirus. The supernatant containing lentivirus was harvested at 48 hours after transfection. Titers were determined on NIH3T3 cells as transducing units using serial dilutions of vector stocks with $8 \mu \mathrm{g} / \mathrm{mL}$ polybrene (Sigma Chemical).

PUER cells (21) are murine hematopoietic precursor cells that have been retrovirally transduced to express $\mathrm{Pu} .1$ fused to the estrogen receptor. To knock down Dnmt1 in cells, murine PUER cells were grown in phenol red-free Iscove's modified Eagle's medium with $10 \%$ fetal bovine serum, $2.5 \mathrm{ng} / \mathrm{mL}$ mouse interleukin-3 (IL-3), $1 \mu \mathrm{g} / \mathrm{mL}$ puromycin, $55 \mu \mathrm{mol} / \mathrm{L} \beta$-mercaptoethanol, $1 \%$ penicillin/streptomycin at $37^{\circ} \mathrm{C}$ in a humidified atmosphere with $5 \% \mathrm{CO}_{2}$ in air. The lentivirus-containing supernatant was added to the cell culture at appropriate 4 particles per cell concentration with $8 \mu \mathrm{g} / \mathrm{mL}$ polybrene. Twenty-four hours after infection, $4 \mu \mathrm{g} / \mathrm{mL}$ blasticidin was added to the cell culture for positive clone selection. The blasticidin-resistant cells were analyzed for Dnmt1 expression by real-time quantitative PCR (RQ-PCR) and Western blot. Control cells were PUER transduced with empty vector (PUER control).
Addition of 4-hydroxytamoxifen (OHT) to PUER triggers their terminal differentiation into macrophages (21). Differentiation status was analyzed by (a) presence of adherent cells by light microscopy, $(b)$ morphologic changes in Giemsa stained cytospin preparations, and (c) c-Kit (eBioscience) and F4/80 (eBioscience) expression by flow cytometry. Cell analysis was done on a Coulter Epics XL-MCL flow cytometer equipped with CXP software (Beckman-Coulter).

\section{Cell fractionation and protein extraction}

Approximately 5 million PUER control and PUER shDnmt1 cells were used to prepare cell lysates. After removal of the medium, cells were transferred to $15-\mathrm{mL}$ conical tubes and washed twice with $5 \mathrm{~mL}$ of ice-cold $1 \times$ PBS. Cells were resuspended in $500 \mu \mathrm{L}$ of $1 \times$ hypotonic buffer containing $10 \mathrm{mmol} / \mathrm{L} \mathrm{HEPES,} 1.5 \mathrm{mmol} / \mathrm{L} \mathrm{MgCl}_{2}$, $10 \mathrm{mmol} / \mathrm{L} \mathrm{KCl}, 0.5 \mathrm{mmol} / \mathrm{L} \mathrm{DTT}, 10 \mathrm{mmol} / \mathrm{L}$ phenylmethylsulfonyl fluoride, and protease inhibitor cocktail (Sigma-Aldrich, A8340) and incubated for 10 minutes on ice. Twenty microliters of $10 \%$ NP40 were added to cell suspensions to break the cell membrane. After another 10-minute incubation on ice, cell suspensions were centrifuged at $500 \times g$ for 10 minutes. The supernatant was transferred to clean 1.5-mL Eppendorf tubes and labeled as the cytoplasm fraction. Nuclear pellets were washed twice with ice-cold water and resuspended in $50 \mu \mathrm{L}$ of $50 \mathrm{mmol} / \mathrm{L}$ Tris-HCl (pH 8.0), $1 \mathrm{mmol} / \mathrm{L} \mathrm{MgCl}_{2}$, $10 \mathrm{mmol} / \mathrm{L}$ phenylmethylsulfonyl fluoride, protease inhibitor cocktail (Sigma-Aldrich, A8340), and DNase 1 (Sigma-Aldrich, D5915, final concentration of 50 units). The nuclear suspensions were incubated on ice for $30 \mathrm{~min}-$ utes with vigorous vortexing every 5 minutes. At the end of incubation, $50 \mu \mathrm{L}$ of protein extraction buffer containing $4 \%$ SDS, $10 \mathrm{mmol} / \mathrm{L} \mathrm{DTT,} \mathrm{20 \%} \mathrm{glycerol} \mathrm{in} 50 \mathrm{mmol} / \mathrm{L} \mathrm{Tris-HCl}$ were added and the mixture was sonicated on ice and centrifuged at 140,000 rpm for 5 minutes. The supernatant containing nuclear proteins was transferred to clean tubes and protein concentration was determined by bicinchoninic acid assay.

\section{One-dimensional SDS-PAGE and Western blotting}

Approximately $50 \mu \mathrm{g}$ of cytoplasmic and nuclear protein extracts from PUER control and PUER shDnmt1 cells, together with molecular weight markers, were subjected to one-dimensional SDS-PAGE on $4 \%$ to $12 \%$ gradient gels (Invitrogen). After electrophoresis per manufacturer's manual (Invitrogen), proteins were transferred onto polyvinylidene difluoride membranes (Millipore) at 35-V constant voltage for 1 hour using an Invitrogen semidry blotting apparatus. Western analyses of polyvinylidene difluoride membranes used established protocols and rabbit monoclonal anti-Pu.1 (Cell Signaling, \#2258) and anti- $\beta$-actin peroxidase (Sigma- Aldrich, A3854).

\section{Real-time PCR}

mRNA levels were assayed using RQ-PCR. Briefly, total cellular RNA was isolated from $5 \times 10^{5}$ cells using RNeasy 
Plus (Qiagen) according to the manufacturer's protocol. For cDNA synthesis, after a denaturation step of 5 minutes at $70^{\circ} \mathrm{C}, 1 \mu \mathrm{g}$ of RNA was reverse transcribed to singlestranded cDNA using a mix of random hexamers and oligo-dT primers and Moloney murine leukemia virus reverse transcriptase for first-strand synthesis (Promega). Real-time PCR was done with Real-time PCR Master Mix containing SYBR Green I and hotstart Taq DNA polymerase (Takara). GAPDH was amplified as control. Primer sequences are available on request. Real-time detection of the emission intensity of SYBR Green bound to double-stranded DNA will be detected using the iCycler instrument (Bio-Rad). Data are reported as relative expression value, which was determined by raising 2 to the power of the negative value of $\delta \delta C_{T}$ for each sample.

\section{Treatment of cells with decitabine}

Decitabine stock solution $(5 \mathrm{mmol} / \mathrm{L})$ was generated by reconstituting lyophilized decitabine in $100 \%$ methanol. Stock solution was stored at $-20^{\circ} \mathrm{C}$ for up to 3 weeks. Working solution was generated by diluting the stock solution 1:100 in PBS immediately before addition to the cells at a further dilution as per the intended final concentration. Similar amounts of methanol are added to untreated control cells. Cells were treated with decitabine $(0.5 \mu \mathrm{mol} / \mathrm{L})$ with timings as designated per the text and figure legends.

\section{Normal cord blood samples}

Umbilical cord blood was collected during normal fullterm deliveries following written informed consent of the mother on a Case Institutional Review Board-approved protocol.

\section{Isolation of $\mathrm{CD}^{+} 4^{+}$cells}

$\mathrm{CD} 4^{+}$cells from umbilical cord blood were immunopositively purified using a magnetic cell sorting system, CD34 MicroBead Kit (Miltenyi Biotec), according to the manufacturer's instructions. The purity of the CD $34^{+}$ population (ranged typically from $95 \%$ to $99 \%$ ) was determined by immunolabeling with FITC-conjugated monoclonal antibodies against CD34 (clone 581, Beckman Coulter) that reacted with an epitope other than the antibody used for separation.

\section{Human hematopoietic cell culture and clonogenic progenitor assays}

CD $34^{+}$selected normal human hematopoietic cells were cultured in Iscove's modified Dulbecco's medium supplemented with $10 \%$ fetal bovine serum and $10 \mathrm{ng} / \mathrm{mL}$ of the following human cytokines: stem cell factor (SCF), FLT3 ligand (FLT3), thrombopoietin (TPO), IL-3, and IL-6. Cells were treated once with $0.5 \mu \mathrm{mol} / \mathrm{L}$ decitabine either concurrently with the addition of $50 \mathrm{ng} / \mathrm{mL}$ G-CSF or 6 hours after $50 \mathrm{ng} / \mathrm{mL}$ G-CSF. Cells were harvested from liquid culture on day 9 and plated into methylcellulose at a concentration of 20,000 cells $/ \mathrm{mL}$ of semisolid medium (Methocult, Stem Cell Technologies) supple- mented with SCF (50 ng/mL), granulocyte macrophage colony-stimulating factor (10 ng/mL), IL-3 (10 ng/mL), and erythropoietin ( 3 units $/ \mathrm{mL}$ ). Colonies were counted and photographed on day 12 of semisolid culture.

\section{Results}

\section{Dnmt1 depletion by shRNA prevents Pu.1-induced HoxB4 repression and differentiation}

Hematopoietic cell fate is driven by key transcription factors such as HOXB4, which promotes hematopoietic stem cell self-renewal (22), and the lineage-specifying factor PU.1, which is required for macrophage and B-cell lineage commitment and differentiation (reviewed in ref. 23). We wished to examine if Dnmt1 is required for the repression of HoxB4 and other stem cell-associated genes by Pu.1. PUER cells are murine Pu.1 $1^{-/-}$hematopoietic precursors that have been transduced with a retroviral vector to express Pu.1 fused to the estrogen receptor (21). In cell culture with murine IL-3 (mIL-3), PUER cells self-renew indefinitely. Addition of OHT to these cells (as an estrogen agonist) causes functional reintroduction of Pu.1 by translocation into the nucleus and triggers terminal macrophage differentiation (21).

Although not normal hematopoietic stem cells, PUER, self-renewing in mIL3, express high levels of the selfrenewal promoting factor HoxB4 and other genes that are expressed at high levels in stem cells, Bmi-1 and c-Kit. After addition of OHT, there is repression of HoxB4, $B m i-1$, and $c-K i t$, followed by activation of genes expressed at high levels in macrophages [macrophage colony stimulating factor receptor (Mcsfr), granulocyte monocyte colony stimulating factor (Gmcsfr), and F4/80; Fig. 1A]. These expression changes were accompanied by acquisition of morphologic changes of macrophage differentiation (increase in cell size, decrease in nuclear-cytoplasmic ratio, clumping of nuclear chromatin, adherence to culture plates, and cytoplasmic vacuolization; Fig. 1A).

Dnmt1 was knocked down in PUER by lentiviral transduction of shRNA (PUER shDnmt1). Control cells were PUER transduced with empty vector (PUER control). Knockdown of Dnmt1 by shDnmt1 was $>70 \%$ by Western blot (Fig. 1B).

Addition of OHT to shDnmt1 cells caused Pu.1-ER to be rapidly translocated from the cytoplasm into the nucleus at high levels (Fig. 1C).

OHT-treated control cells rapidly repressed expression of stem cell genes HoxB4, Bmi-1, and c-Kit; however, OHTtreated shDnmt1 cells continued to express high levels of these stem cell genes (Fig. 1D). OHT-treated control cells activated expression of differentiation genes Mcsfr, Gmcsfr, and F4/80; however, these genes remained relatively repressed in OHT-treated shDnmt1 cells (Fig. 1D).

OHT-treated control cells displayed extensive morphologic changes of macrophage differentiation (Fig. 1D). In contrast, OHT-treated shDnmt1 cells continued to display morphologic immaturity, with most cells resembling untreated shDnmt1 cells (Fig. 1D). 


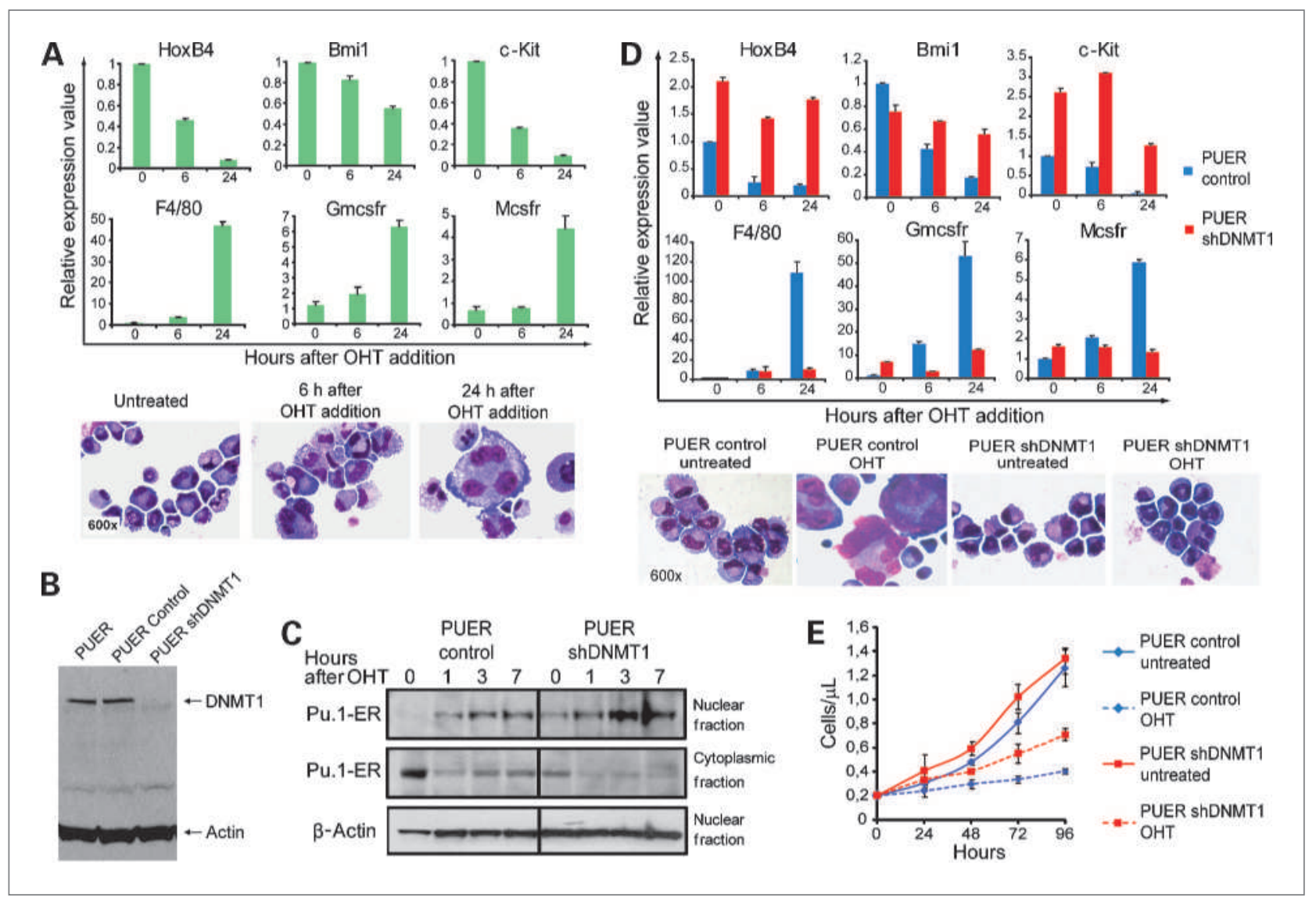

Figure 1. Dnmt1 depletion by shRNA prevents Pu.1-mediated repression of stem cell-associated genes and allows continued self-renewal in the presence of nuclear Pu.1. PUER are Pu. $1^{-/-}$murine hematopoietic precursors retrovirally transduced to express Pu.1 fused to the estrogen receptor. OHT $(100 \mathrm{nmol} / \mathrm{L})$ translocates Pu. 1 to the nucleus and triggers macrophage differentiation. A, after addition of OHT, there is repression of stem cell-associated genes HoxB4, Bmi-1, and c-Kit, followed by activation of genes associated with macrophage differentiation, Mcsfr, Gmcsfr, and F4/80. Giemsa-stained cytospin preparations $48 \mathrm{~h}$ after addition of OHT. Gene expression measured by RQ-PCR; average of six independent experiments. B, Dnmt1 was knocked down in PUER by lentiviral transduction of shRNA (PUER shDnmt1) with PUER transduced with empty vector as control (PUER control). Dnmt1 measured by Western blot. C, addition of $\mathrm{OHT}$ to shDnmt1 cells caused Pu.1-ER to be rapidly translocated from the cytoplasm into the nucleus at high levels. Pu.1-ER measured by Western blot in nuclear and cytoplasmic fractions. Actin in nuclear fraction. D, OHT-treated shDnmt1 cells continued to express high levels of stem cell-associated genes and low levels of differentiation genes. Gene expression by RQ-PCR; average of three independent experiments. Giemsa-stained cytospin preparations $48 \mathrm{~h}$ after OHT addition. E, OHT-treated shDnmt 1 cells proliferated at a lower rate than shDnmt 1 cells without $\mathrm{OHT}$; however, the cells continued to exponentially expand at a significantly higher rate than OHT-treated control cells. Cell counts by automated cell counter; average of three independent experiments. Bars, SE.

OHT treatment substantially decreased the proliferation of control cells. OHT treatment also decreased the proliferation of shDnmt1 cells, but not to the same extent as the decrease in proliferation of control cells (OHT-treated shDnmt1 cells continued to expand exponentially; Fig. 1E).

Therefore, depletion of Dnmt1 prevented Pu.1-mediated repression of HoxB4 and other stem cell genes, prevented differentiation, and allowed hematopoietic precursor self-renewal in the presence of nuclear Pu.1.

\section{Maintenance of hematopoietic precursor self- renewal by decitabine requires timing of treatment to prevent Pu.1-mediated stem cell gene repression}

To further develop this observation, decitabine, the cytosine analogue that binds and depletes Dnmt1, was added to the PUER cells concurrent with the introduction of Pu.1 (concurrent with OHT) or 6 hours after introduction of Pu.1 (6 hours after OHT), that is, after the repression of HoxB4 had occurred.

OHT treatment of PUER caused rapid repression of the stem cell genes HoxB4, Bmi-1, and c-Kit, coupled with activation of the differentiation genes F4/80, Gmcsfr, and Mcsfr. Decitabine addition concurrent with OHT attenuated the repression of the stem cell genes and the activation of the differentiation genes. Decitabine addition 6 hours after OHT did not significantly impede stem cell gene repression or the subsequent differentiation gene activation (Fig. 2A).

OHT treatment of PUER caused rapid macrophage differentiation. Decitabine concurrent with OHT largely prevented morphologic differentiation, with most cells resembling cells without OHT. Decitabine 6 hours 
after OHT resulted in more extensive changes of macrophage differentiation than with OHT alone (Fig. 2A).

OHT treatment of PUER caused a substantial decrease in proliferation compared with untreated cells. Decitabine concurrent with OHT also decreased cell proliferation compared with untreated cells, but not to the same extent as OHT alone. Decitabine 6 hours after OHT terminated cell proliferation (Fig. 2B).

OHT treatment of PUER caused a decrease in c-Kit protein expression and an increase in F4/80 protein expression as measured by flow cytometry. Decitabine concurrent with OHT significantly attenuated the decrease in c-Kit expression and the increase in F4/80 expression produced by OHT alone. Decitabine 6 hours after OHT resulted in C-Kit and F4/80 expression changes that resembled OHT alone (Fig. 2B).

Therefore, the ability of decitabine to maintain selfrenewal of hematopoietic precursors in the presence of nuclear Pu.1 depends on timing of decitabine treatment in relationship to the Pu.1 differentiation stimulus.

\section{Maintenance of human hematopoietic precursor self-renewal in the presence of G-CSF requires timing of decitabine treatment to prevent stem cell-associated gene repression by G-CSF}

Previously, we and others showed that treating human $\mathrm{CD}_{3} 4^{+}$hematopoietic stem and progenitor cells with decitabine maintains or promotes their self-renewal in vitro such that decitabine can be used for ex vivo expansion of hematopoietic stem and progenitor cells $(8,9,24)$.

Human $\mathrm{CD}_{3} 4^{+}$cells express high levels of HOXB4 and other factors known to be associated with stem cell self-renewal such as the stem cell factor receptor (c-KIT) and the polycomb protein BMI-1 (Fig. 3A). Addition of the myeloid differentiation-promoting cytokine G-CSF to these cells results in rapid repression

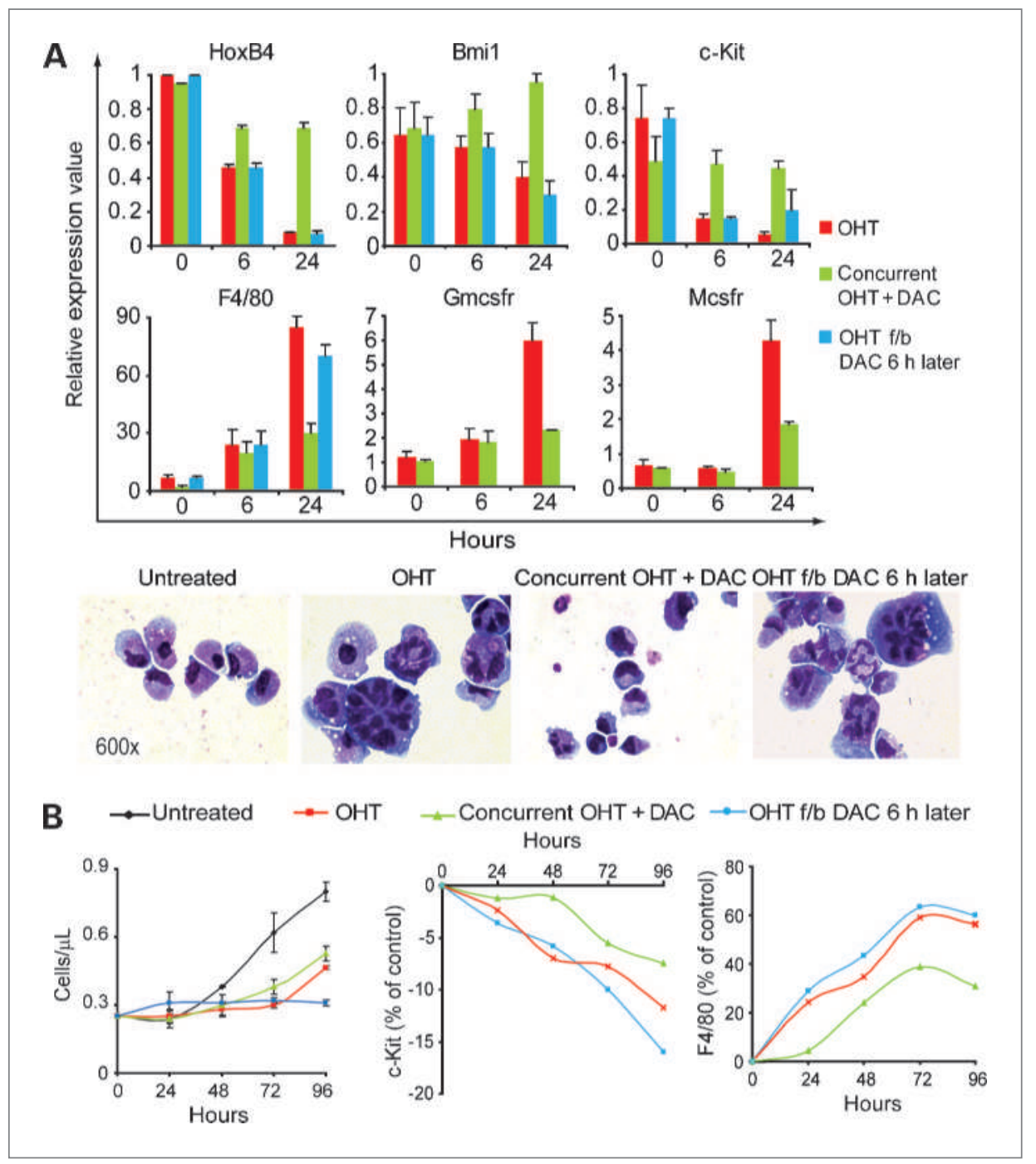

Figure 2. Maintenance of hematopoietic precursor (PUER) self-renewal by $0.5 \mu \mathrm{mol} / \mathrm{L}$ decitabine (DAC) requires timing of decitabine treatment to prevent Pu.1-mediated stem cell gene repression. A, decitabine addition concurrent with $\mathrm{OHT}$ (Pu.1), but not $6 \mathrm{~h}$ after $\mathrm{OHT}$, attenuated the repression of stem cell genes (HoxB4, Bmi-1, and C-Kit) and the activation of differentiation genes (F4/80, Gmcsfr, and Mcsfr) compared with $\mathrm{OHT}$ alone. Gene expression by RQ-PCR; average of four independent experiments. Giemsa-stained cytospin preparations $48 \mathrm{~h}$ after $\mathrm{OHT}$. $\mathrm{B}$, decitabine addition concurrent with OHT (Pu.1) attenuated the decrease in proliferation and the protein expression changes seen with $\mathrm{OHT}$ alone. Cells counts by automated cell counter; average of three independent experiments. Bars, SE. c-Kit and F4/80 protein expression measured by flow cytometry. 


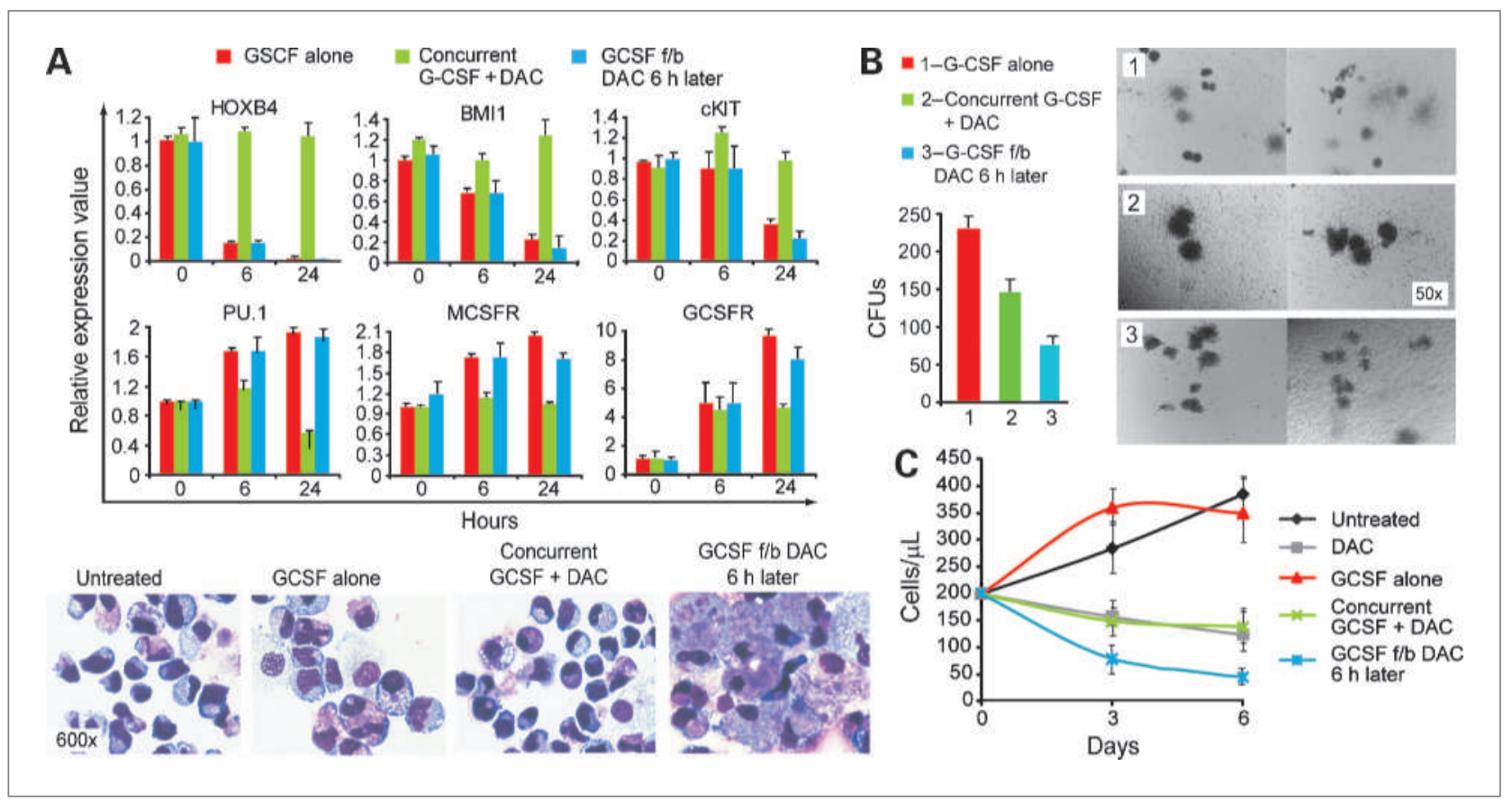

Figure 3. Cell fate in response to $0.5 \mu \mathrm{mol} / \mathrm{L}$ decitabine treatment varies with timing in relationship to G-CSF differentiation stimulus. Human CD34 hematopoietic precursors were cultured with SCF, TPO, FLT3, IL-3, and IL-6 with or without G-CSF and decitabine. A, DAC concurrent with G-CSF, but not $6 \mathrm{~h}$ after G-CSF, significantly attenuated the repression of HOXB4, BMI1, and C-KIT and attenuated the activation of PU.1, MCSFR, and GCSFR, compared with G-CSF alone. Gene expression by RQ-PCR; average of four independent experiments. Giemsa-stained cytospin preparations on day 7 after G-CSF addition. B, DAC addition concurrent with G-CSF decreased the number of progenitors but increased the size of colonies formed. DAC $6 \mathrm{~h}$ after G-CSF decreased both the number and the size of colonies. Cells harvested from liquid culture $9 \mathrm{~d}$ after addition of G-CSF. Cells plated into methylcellulose at 20,000 cells $/ \mathrm{mL}$ of semisolid medium. Colonies were counted and photographed on day 12 of semisolid culture ( $n=4$ ). C, DAC concurrent with G-CSF decreased cell proliferation compared with G-CSF alone, but not to the same extent as DAC $6 \mathrm{~h}$ after G-CSF. Cell counts by automated cell counter; average of four independent experiments. Bars, SE.

of the stem cell genes HOXB4, BMI-1, and $c$-KIT, coupled with activation of the differentiation genes PU.1, $M C S F R$, and G-CSF receptor (G-CSFR; Fig. 3A). Addition of decitabine concurrent with G-CSF significantly attenuated repression of the stem cell genes and attenuated activation of the differentiation genes compared with G-CSF alone. Addition of decitabine 6 hours after G-CSF did not impede stem cell gene repression or differentiation gene activation compared with G-CSF alone (Fig. 3A).

G-CSF induced morphologic differentiation of the cells (increased cell size, decreased nuclear-cytoplasmic ratio, chromatin clumping, and increased granulation; Fig. 3A). Decitabine addition concurrent with G-CSF largely prevented the morphologic differentiation seen with G-CSF alone (Fig. 3A). In contrast, decitabine addition 6 hours after G-CSF produced more extensive morphologic changes of differentiation compared with cells treated with G-CSF alone (Fig. 3A).

Decitabine addition concurrent with G-CSF decreased the number of progenitors present on day 9 (9 days after addition of G-CSF) of liquid culture (progenitors were assayed by plating cells harvested from liquid culture into semisolid media) compared with G-CSF alone. However, the colonies formed were larger, consistent with more immature character of the progenitors (Fig. 3B). In contrast, decitabine addition 6 hours after G-CSF (after HOXB4 repression and PU.1 activation) produced a greater decrease in colony number (decreased progenitors) and colony size (increased maturity of progenitors) compared with cells treated with concurrent decitabine and G-CSF or G-CSF alone (Fig. 3B).

Decitabine addition concurrent with G-CSF decreased cell proliferation compared with G-CSF alone, but not to the same extent as decitabine addition 6 hours after G-CSF (Fig. 3C).

Therefore, the effects of decitabine treatment on human hematopoietic cell fate depend on timing of treatment in relationship to the cytokine differentiation stimulus.

\section{Discussion}

The repression of HOXB4 and other stem cell genes that occurs early after a differentiation stimulus is necessary for differentiation to proceed. This suggests a mechanistic basis for the observation that drugs that antagonize transcription repression can increase hematopoietic stem cell self-renewal (8-10, 19, 20, 24-26). DNMT1 depletion by decitabine, before or concurrent with a differentiationinducing stimulus, by preventing the repression of stem 
cell genes by the differentiation stimulus, maintains stem cell self-renewal. Consistent with the proposed mechanism, treatment with decitabine shortly after a differentiation stimulus (after transition through the early repression phase) does not maintain self-renewal and instead augments differentiation.

An alternative model to explain the ability of decitabine to increase hematopoietic stem cell self-renewal is that decitabine treatment to deplete DNMT1 directly reactivates a stem cell program in the treated cells. The different cell fate produced by treating cells with decitabine before versus after a differentiation stimulus is more consistent with the proposed model than this alternative. Emphasizing that the cell fate consequences of decitabine treatment are cell context dependent, decitabine treatment induces terminal differentiation of leukemia cells (1-5), including the demonstrably self-renewing subset of leukemia-initiating cells, ${ }^{4}$ in contrast to its effect on normal hematopoietic stem cells.

Another question is whether the differentiation-altering effects of decitabine can be attributed to DNMT1 depletion rather than to other effects of the drug. Decitabine is a cytosine analogue, and therefore, as per the class effect of nucleoside analogues, it can induce DNA damage and cytotoxicity. However, decitabine is rapidly cleaved and degraded by hydrolysis (27). Hence, decitabine is substantially less efficient at impeding DNA replication machinery and terminating DNA strand elongation than an equimolar concentration of cytosine arabinoside (ara-C), the cytosine analogue that is the mainstay of cytotoxic acute myelogenous leukemia therapy $(28,29)$. We have shown that the concentrations of decitabine used here deplete DNMT1 without causing measurable DNA damage or apoptosis in normal human hematopoietic precursors. ${ }^{4}$ Others have shown that decitabine depletes the maintenance DNA methyltransferase DNMT1 (11), but not the de novo DNA methyltransferases DNMT3A and DNMT3B (11), consistent with the S-phase specificity of decitabine. The similar shifts in differentiation produced by HDAC inhibitors and decitabine are also consistent with a mechanism that relates to antagonism of transcription repression. Finally, conditional deletion of DNMT1 by genetic methods shows that it has a role in regulating hematopoietic cell fate. In a murine model of inducible knockout of Dnmt1 in adult mice, defects in the hematopoietic stem cell compartment and in progenitors suggested that Dnmt1 is required for hematopoietic stem cell self-renewal and differentiation (30). In another genetic study, Dnmt1 was partially depleted using a hypomorphic Dnmt1 allele (31). An increase in hematopoietic stem cells and deficiencies in lineagecommitted progenitors were noted (31).

Although the murine conditional Dnmt1 knockout models show a role for Dnmt1 in hematopoietic differentiation, these genetic models differ from DNMT1 depletion by decitabine treatment in important respects: DNMT1 depletion by decitabine is transient, with recovery of DNMT1 protein in normal hematopoietic precursors to close to pretreatment levels within 96 hours of decitabine exposure ${ }^{5}$; the transient DNMT1 depletion by decitabine, although substantial, is not complete $(3,11)$; DNMT1 depletion by decitabine is S-phase dependent and is therefore more extensive in actively cycling cells such as hematopoietic precursors than in supporting tissue such as stroma.

The findings here suggest that DNMT1, a key chromatin modifying enzyme involved in transcription repression, is required for the early repression of stem cell genes that occurs in response to a differentiation stimulus. This repression step is necessary for differentiation to proceed to the next level. The varied cell fate consequences of decitabine treatment reflect the differentiation phase- and cell context-dependent role of the DNMT1 molecular target of therapy.

\section{Disclosure of Potential Conflicts of Interest}

Y. Saunthararajah: previous consultant for HemaQuest, a company developing treatments for blood diseases such as thalassemia and sickle cell disease. No other potential conflicts of interest were disclosed.

\section{Acknowledgments}

We thank Mary Laughlin and Nick Greco at the Abraham J and Phyllis Katz Cord Blood Foundation and Cleveland Cord Blood Center for the kind gift of cord blood samples, and Dr. Brian Bolwell for Departmental support and resources.

\section{Grant Support}

Scott Hamilton CARES Foundation, NIH grants U54HL090513 and 1R01CA138858 and Department of Defense grant PR081404 (Y. Saunthararajah); NIH grants R01HL082983, U54RR019391, and K24HL077522, the AA\&MDS Foundation, and the Robert Duggan Cancer Research Foundation (J.P. Maciejewski).

The costs of publication of this article were defrayed in part by the payment of page charges. This article must therefore be hereby marked advertisement in accordance with 18 U.S.C. Section 1734 solely to indicate this fact.

Received 02/26/2010; accepted 04/12/2010; published OnlineFirst $05 / 25 / 2010$.

\section{References}

1. Jones PA, Taylor SM. Cellular differentiation, cytidine analogs and DNA methylation. Cell 1980;20:85-93.

2. Pinto A, Attadia V, Fusco A, Ferrara F, Spada OA, Di Fiore PP. 5Aza-2'deoxycytidine induces terminal differentiation of leukemic

blasts from patients with acute myeloid leukemias. Blood 1984; 64:922-9.

3. Creusot F, Acs G, Christman JK. Inhibition of DNA methyltransferase and induction of Friend erythroleukemia cell differentiation by 
5-azacytidine and 5-aza-2'-deoxycytidine. J Biol Chem 1982;257: 2041-8.

4. Nowak D, Stewart D, Koeffler HP. Differentiation therapy of leukemia: 3 decades of development. Blood 2009;113:3655-65.

5. Niitsu N, Hayashi Y, Sugita K, Honma Y. Sensitization by 5-aza-2'deoxycytidine of leukaemia cells with MLL abnormalities to induction of differentiation by all-trans retinoic acid and 1a,25-dihydroxyvitamin D3. Br J Haematol 2001;112:315-26.

6. Saunthararajah $Y$, Hillery CA, Lavelle D, et al. Effects of 5-aza-2adeoxycytidine on fetal hemoglobin levels, red cell adhesion, and hematopoietic differentiation in patients with sickle cell disease. Blood 2003;102:3865-70.

7. Saunthararajah $\mathrm{Y}$, Molokie $\mathrm{R}$, Saraf $\mathrm{S}$, et al. Clinical effectiveness of decitabine in severe sickle cell disease. $\mathrm{Br} \mathrm{J}$ Haematol 2008;141: 126-9.

8. Milhem M, Mahmud N, Lavelle D, et al. Modification of hematopoietic stem cell fate by 5 aza 2 'deoxycytidine and trichostatin A. Blood 2004;103:4102-10.

9. Suzuki M, Harashima A, Okochi A, et al. 5-Azacytidine supports the long-term repopulating activity of cord blood CD34 ${ }^{+}$cells. Am J Hematol 2004;77:313-5.

10. Chung YS, Kim HJ, Kim TM, et al. Undifferentiated hematopoietic cells are characterized by a genome-wide undermethylation dip around the transcription start site and a hierarchical epigenetic plasticity. Blood 2009;114:4968-78.

11. Ghoshal K, Datta J, Majumder S, et al. 5-Aza-deoxycytidine induces selective degradation of DNA methyltransferase 1 by a proteasomal pathway that requires the KEN box, bromo-adjacent homology domain, and nuclear localization signal. Mol Cell Biol 2005;25:4727-41.

12. Zucker KE, Riggs AD, Smith SS. Purification of human DNA (cytosine-5-)-methyltransferase. J Cell Biochem 1985;29:337-49.

13. Kohn KW, Aladjem MI, Weinstein JN, Pommier Y. Chromatin challenges during DNA replication: a systems representation. Mol Biol Cell 2008;19:1-7.

14. Wang J, Hevi S, Kurash JK, et al. The lysine demethylase LSD1 (KDM1) is required for maintenance of global DNA methylation. Nat Genet 2009;41:125-9.

15. Haaf $T$. The effects of 5-azacytidine and 5-azadeoxycytidine on chromosome structure and function: implications for methylationassociated cellular processes. Pharmacol Ther 1995;65:19-46.

16. Kosugi $H$, Towatari $M$, Hatano $S$, et al. Histone deacetylase inhibitors are the potent inducer/enhancer of differentiation in acute myeloid leukemia: a new approach to anti-leukemia therapy. Leukemia 1999;13:1316-24.

17. Wang J, Saunthararajah Y, Redner RL, Liu JM. Inhibitors of histone deacetylase relieve ETO-mediated repression and induce differentiation of AML1-ETO leukemia cells. Cancer Res 1999;59:2766-9.

18. Desimone J. Approaches to the reactivation of hemoglobin $\mathrm{F}$ as a treatment for sickle cell disease. Clin Adv Hematol Oncol 2004;2: 23-4.

19. Bug G, Gul H, Schwarz K, et al. Valproic acid stimulates proliferation and self-renewal of hematopoietic stem cells. Cancer Res 2005;65: 2537-41.

20. De Felice L, Tatarelli C, Mascolo MG, et al. Histone deacetylase inhibitor valproic acid enhances the cytokine-induced expansion of human hematopoietic stem cells. Cancer Res 2005;65:1505-13.

21. Walsh JC, DeKoter RP, Lee HJ, et al. Cooperative and antagonistic interplay between PU.1 and GATA-2 in the specification of myeloid cell fates. Immunity 2002;17:665-76.

22. Sauvageau G, Thorsteinsdottir U, Eaves CJ, et al. Overexpression of HOXB4 in hematopoietic cells causes the selective expansion of more primitive populations in vitro and in vivo. Genes Dev 1995;9: 1753-65.

23. Iwasaki $H$, Akashi K. Myeloid lineage commitment from the hematopoietic stem cell. Immunity 2007;26:726-40.

24. Araki $\mathrm{H}$, Mahmud $\mathrm{N}$, Milhem $\mathrm{M}$, et al. Expansion of human umbilical cord blood SCID-repopulating cells using chromatin-modifying agents. Exp Hematol 2006;34:140-9.

25. Young JC, Wu S, Hansteen G, et al. Inhibitors of histone deacetylases promote hematopoietic stem cell self-renewal. Cytotherapy 2004;6:328-36.

26. Lee $\mathrm{JH}$, Hart SR, Skalnik DG. Histone deacetylase activity is required for embryonic stem cell differentiation. Genesis 2004;38:32-8.

27. Rogstad DK, Herring JL, Theruvathu JA, et al. Chemical decomposition of 5-aza-2'-deoxycytidine (decitabine): kinetic analyses and identification of products by NMR, HPLC, mass spectrometry. Chem Res Toxicol 2009;22:1194-204.

28. Covey JM, D'Incalci M, Tilchen EJ, Zaharko DS, Kohn KW. Differences in DNA damage produced by incorporation of 5-aza-2'-deoxycytidine or 5,6-dihydro-5-azacytidine into DNA of mammalian cells. Cancer Res 1986;46:5511-7.

29. Schermelleh L, Haemmer A, Spada F, et al. Dynamics of Dnmt1 interaction with the replication machinery and its role in postreplicative maintenance of DNA methylation. Nucleic Acids Res 2007;35: 4301-12.

30. Trowbridge JJ, Snow JW, Kim J, Orkin SH. DNA methyltransferase 1 is essential for and uniquely regulates hematopoietic stem and progenitor cells. Cell Stem Cell 2009;5:442-9.

31. Broske AM, Vockentanz L, Kharazi S, et al. DNA methylation protects hematopoietic stem cell multipotency from myeloerythroid restriction. Nat Genet 2009;41:1207-15. 


\section{Molecular Cancer Therapeutics}

\section{Decitabine Maintains Hematopoietic Precursor Self-Renewal by Preventing Repression of Stem Cell Genes by a Differentiation-Inducing Stimulus}

Zhenbo Hu, Soledad Negrotto, Xiaorong Gu, et al.

Mol Cancer Ther 2010;9:1536-1543. Published OnlineFirst May 25, 2010.

Updated version Access the most recent version of this article at: doi:10.1158/1535-7163.MCT-10-0191

Cited articles This article cites 31 articles, 13 of which you can access for free at: http://mct.aacrjournals.org/content/9/6/1536. full\#ref-list-1

Citing articles This article has been cited by 7 HighWire-hosted articles. Access the articles at: http://mct.aacrjournals.org/content/9/6/1536.full\#related-urls

E-mail alerts Sign up to receive free email-alerts related to this article or journal.

Reprints and To order reprints of this article or to subscribe to the journal, contact the AACR Publications Subscriptions Department at pubs@aacr.org.

Permissions To request permission to re-use all or part of this article, use this link http://mct.aacrjournals.org/content/9/6/1536.

Click on "Request Permissions" which will take you to the Copyright Clearance Center's (CCC) Rightslink site. 